\title{
Neutrino Mass from a $d=7$ Effective Operator in a SUSY-GUT Framework
}

\author{
Martin B. Krauss ${ }^{1}$, Davide Meloni ${ }^{2}$, Werner Porod ${ }^{3}$, and Walter Winter ${ }^{4}$ \\ 1,3,4 Institut für Theoretische Physik und Astrophysik, Universität Würzburg, \\ Am Hubland, 97074 Würzburg, Germany \\ ${ }^{2}$ Dipartimento di Matematica e Fisica, Università degli Studi Roma Tre, \\ Via del la Vasca Navale 84, 00146 Roma, Italy
}

October 31,2018

\begin{abstract}
Models, where neutrino mass originates from physics at the $\mathrm{TeV}$ scale and which are potentially testable at the LHC, need additional suppression mechanisms to describe the smallness of neutrino masses. We consider models in which neutrino mass is generated from the $d=7$ operator $L L H_{u} H_{u} H_{d} H_{u}$ in the context of SUSY-GUTs containing an SU(5) subgroup, where the $d=5$ Weinberg operator can be forbidden by a discrete symmetry. That is, we identify the embeddings in GUT multiplets and their consequences for phenomenology and renormalization group evolution. We use a specific example to exemplify the challenges. In this case, additional heavy $d$-quarks are predicted, which are constrained by cosmology, in particular, by big bang nucleosynthesis and direct searches for heavy nuclei. We show that in the NMSSM extension of the model, the discrete symmetry needs to be broken, which can be the origin of deviations from tri-bimaximal mixings. Finally we demonstrate that our example is the only tree level decomposition which is consistent with perturbativity up to the GUT scale and neutrino mass generation by a leading $d=7$ contribution.
\end{abstract}

\section{Introduction}

The recent measurement of $\theta_{13}$ by Daya Bay [1] and RENO [2] has revealed that the last of the three angles of a minimal three-generation model for neutrino physics is relatively large, which opens the window for the discovery of leptonic CP violation. This result exerts pressure on flavor symmetry models (such as based on the permutation groups $A_{4}$ and $S_{4}$ ), as they are generically constructed in a way to give at leading order tri-bimaximal (or bi-maximal) mixing - thus implying $\theta_{13}=0$ (see [3] for an extended review). Corrections from the charged sectors or next-to-leading contributions to the neutrino mass matrix are generally invoked to correct such patterns and make the models compatible with the experimental data.

With the recent discovery of a bosonic resonance $[4,5]$, showing all the characteristics of the SM Higgs boson, a long search might soon come to a successful end. In contrast no conclusive hints for physics beyond the Standard Model (SM) have been found [6-15]. Therefore, large areas of the parameter space of the simplest SUSY models are excluded now and the allowed mass spectra as well as the best fit values to the data are pushed to higher and higher values, see, e.g. $[16,17]$ and Refs. therein. This has lead to an increasing interest in the study of SUSY models which provide new features. For instance, models with light stops and higgsinos can more easily explain the recent findings within the minimal-supersymmetric standard model (MSSM), see e.g. [18-21], while models with broken $R$-parity might be able to hide much better at the LHC [22, 23]. Moreover, the MSSM extension with a singlet Higgs boson [24-29] or an

\footnotetext{
${ }^{1}$ Email: martin.krauss@physik.uni-wuerzburg.de

${ }^{2}$ Email: meloni@fis.uniroma3.it

${ }^{3}$ Email: porod@physik.uni-wuerzburg.de

${ }^{4}$ Email: winter@physik.uni-wuerzburg.de
} 
additional gauge group can easier explain a Higgs boson with a mass of $126 \mathrm{GeV}$ than the MSSM [30-36]. Most of these models will be tested at the LHC after its energy upgrade to $14 \mathrm{TeV}$.

Generic models to describe the smallness of neutrino mass, such as the standard type-I seesaw mechanism [37-40], imply new physics close to the GUT scale. In these seesaw models, one can integrate out the heavy mediators in order to obtain the famous $d=5$ Weinberg operator [41],

$$
\mathcal{L}_{\text {eff }}^{d=5} \propto \frac{1}{\Lambda_{\mathrm{NP}}}\left(\overline{L^{c}} \mathrm{i} \tau^{2} H\right)\left(H \mathrm{i} \tau^{2} L\right)
$$

which leads, after Electroweak Symmetry Breaking (EWSB), to Majorana masses $m_{\nu} \sim v^{2} / \Lambda_{\mathrm{NP}}$ for the neutrinos. Here $L$ and $H$ stand for the SM lepton and Higgs doublets, respectively, $v$ for the Higgs vev, and $\Lambda_{\mathrm{NP}}$ for the new physics scale. For order one couplings, $\Lambda_{\mathrm{NP}}$ points to the GUT scale, which means that these models are not accessible by experiments. In addition, they contain more parameters than there are observables in the neutrino sector.

It is therefore potentially attractive to test neutrino mass models with new particles at the TeV scale, since current and future LHC upgrades can directly discover or constrain these models. In such models, the smallness of the neutrino mass requires (at least) an additional suppression mechanism: Examples are radiative mass generation, where loop suppression factors enter, see Refs. [42-47] for systematic approaches, or models with a small lepton number violating contribution, such as the inverse seesaw mechanism [48] or SUSY with R-parity violation where both aspects enter, see for example [49-58].

In this paper, we focus on another possibility, which has been recently drawing some attention: If the operator in Eq. (1) is forbidden, such as by a discrete symmetry, a higher dimensional operator may dominate [43, 59-70]. Such models can also be combined with supersymmetry, see for example [61, 71]. The simplest possibilities of operators in the MSSM, leading to neutrino mass, might be

$$
\mathcal{L}_{\text {eff }}^{d=2 n+5}=\frac{1}{\Lambda_{\mathrm{NP}}^{d-4}}\left(L L H_{u} H_{u}\right)\left(H_{d} H_{u}\right)^{n}, \quad n=1,2,3, \ldots,
$$

whereas in the NMSSM,

$$
\mathcal{L}_{\mathrm{eff}}^{d=n+5}=\frac{1}{\Lambda_{\mathrm{NP}}^{d-4}}\left(L L H_{u} H_{u}\right)(S)^{n}, \quad n=1,2,3, \ldots
$$

or mixed combinations with Eq. (2) are possible, see Ref. [71] for a detailed discussion. In all these cases, neutrino mass is suppressed by a higher power of $\Lambda_{\mathrm{NP}}$, which means that $\Lambda_{\mathrm{NP}}$ may be potentially the TeV scale.

The simplest possibility which works both in the MSSM and the NMSSM is the $d=7$ operator

$$
\mathcal{L}_{\mathrm{eff}}^{d=7}=\frac{1}{\Lambda_{\mathrm{NP}}^{3}}\left(L L H_{u} H_{u}\right)\left(H_{d} H_{u}\right)
$$

This operator is interesting for two reasons: First of all, it already implies a substantial suppression of neutrino mass, more than the $d=6$ operator in Eq. (3) (for $n=1$ ). And second, in the NMSSM framework the concept of neutrino mass generation can be extended to higher $(d>7)$ dimensions, $c f$., Eq. (2), whereas Eq. (3) as leading contribution to neutrino mass is in fact limited to $n \leq 2$, and operators with mixed combinations of $S$ and $\left(H_{d} H_{u}\right)$ cannot exceed $d=7$ as well. ${ }^{1}$ Therefore, we focus on Eq. (4) in this study.

In Ref. [71], we have studied a particular example for the generation of this operator which leads potentially to interesting signals at the LHC. In this paper we go a step further and investigate how potential GUT completions of such models look like and in which $S U(5)$ representation the corresponding mediators would reside in. This is motivated by the fact that some of the newly postulated particles are charged under the SM gauge group and, thus, would destroy the successful MSSM prediction for gauge

\footnotetext{
${ }^{1}$ The reason for that in the NMSSM is that the $\hat{S} \hat{H}_{u} \hat{H}_{d}$ and $\hat{S}^{3}$ terms have to be allowed by the discrete symmetry, which implies that certain lower dimensional operators leading to neutrino mass cannot be forbidden [71].
} 


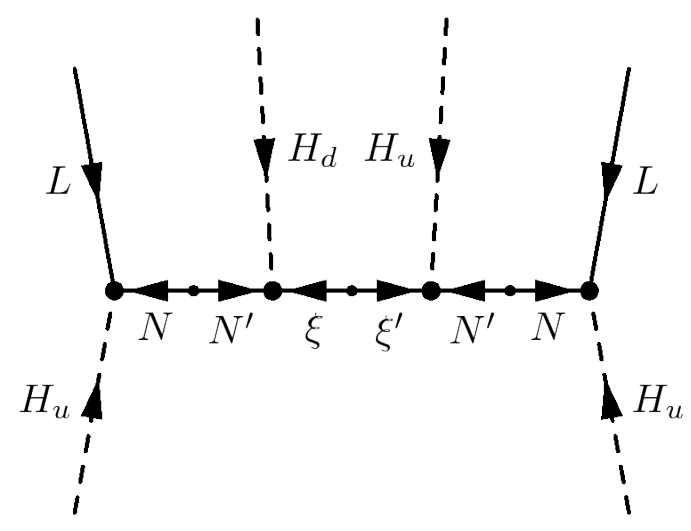

Figure 1: Example for neutrino mass chosen in section 2. Here $N$ and $N^{\prime}$ are fermion singlets, and $\xi$ and $\xi^{\prime}$ are fermion doublets.

coupling unification [72-78]. This is similar to the case of the seesaw type II and type III mechanisms in which the corresponding $S U(2)$ triplets have to be included in complete $S U(5)$ multiplets leading to interesting effects for collider physics and the abundance of the relic dark matter density [79-88]. The requirement to obtain gauge coupling unification implies that not only the mediators for the higher dimensional neutrino mass operators have masses at the $\mathrm{TeV}$ scale, but also the members of the corresponding $S U(5)$ multiplets. However, renormalization group effects can lead to a sizable mass splitting in particular for those particles charged under $S U(3)_{C}$.

The plan of this paper is as follows: we start with the model presented in Ref. [71] and discuss its GUT completion considering both, the MSSM Higgs sector as well as the NMSSM Higgs sector. As we will see, we have to embed heavy $S U(2)$ leptons, required by the model, into $S U(5)$ five-plet representations, which implies the prediction of new heavy d-quarks at the $\mathrm{TeV}$-scale. In order to avoid the $\mu$-problem of the MSSM, we discuss the NMSSM implementation in section 3 and its challenges, as well as we propose an origin of deviations from tri-bimaximal mixings in this approach. In section 4, we review the LHC and cosmological constraints on the heavy d-quarks, and propose fast enough decay modes compatible with the model. In section 5 we systematically discuss the GUT completions of the possibilities for neutrino mass from the higher dimensional operator suggested in [71]. Here we show that several of them would imply non-perturbative gauge couplings already significantly below the GUT scale. We constrain ourselves to the $S U(5)$ case as it is also a subgroup of larger groups such as $S O(10)$ or $E_{6}$. Finally in section 6 we summarize and draw our conclusions.

\section{A low energy model for a realization of a $d=7$ neutrino mass oper- ator and its GUT completion}

In Ref. [42], it has been demonstrated that at tree-level, there are exactly three decompositions of the Weinberg operator, which are known as type I, type II, and type III seesaw mechanisms, respectively. Similarly, the $d=7$ operator in Eq. (4) can be decomposed systematically at tree-level, as discussed in Ref. [65] for the Two Higgs Doublet Model and in Ref. [71] for SUSY. As a side remark, note that supersymmetry constrains the possible decompositions of these operators in contrast to the Weinberg operator, where all three possibilities have also a supersymmetric realization.

Here we will first recall a particular decomposition of a $d=7$ operator, which has been already presented in Ref. [71], and discuss its GUT completion to exemplify the challenges one encounters. The tree-level diagram for the leading contribution to neutrino mass is shown in Fig. 1. The corresponding superpotential is, at the electroweak scale, given by

$$
W=W_{\mathrm{NMSSM}}+Y_{N} \hat{N} \hat{L} \cdot \hat{H}_{u}-\kappa_{1} \hat{N}^{\prime} \hat{\xi} \cdot \hat{H}_{d}+\kappa_{2} \hat{N}^{\prime} \hat{\xi}^{\prime} \cdot \hat{H}_{u}+m_{N} \hat{N} \hat{N}^{\prime}+m_{\xi} \hat{\xi} \cdot \hat{\xi}^{\prime}
$$


where the mediators $N$ and $N^{\prime}$ are SM singlets and $\xi$ and $\xi^{\prime}$ are $S U(2)_{L}$ doublets carrying hypercharge $1 / 2$ and $-1 / 2$, respectively. After electroweak symmetry breaking the mass matrix for the neutral weakly interacting fermions reads schematically in the basis $f^{0}=\left(\nu, N, N^{\prime}, \xi^{0}, \xi^{\prime 0}\right)$ as

$$
M_{f}^{0}=\left(\begin{array}{ccccc}
0 & Y_{N} v_{u} & 0 & 0 & 0 \\
Y_{N} v_{u} & 0 & m_{N} & 0 & 0 \\
0 & m_{N} & 0 & \kappa_{1} v_{d} & \kappa_{2} v_{u} \\
0 & 0 & \kappa_{1} v_{d} & 0 & m_{\xi} \\
0 & 0 & \kappa_{2} v_{u} & m_{\xi} & 0
\end{array}\right),
$$

where we have neglected all flavor indices. By integrating out the heavy fields one obtains an effective mass matrix for the three SM neutrinos

$$
m_{\nu}=v_{u}^{3} v_{d} Y_{N}^{2} \frac{\kappa_{1} \kappa_{2}}{m_{\xi} m_{N}^{2}}
$$

where the couplings carry a flavor index. As has been demonstrated in Ref. [71], one successfully explains neutrino masses and mixings within this model by an appropriate choice of fermion generations and coupling matrices. In addition, one can easily see from Eq. (6) that the neutral fermion mass matrix reduces to an inverse seesaw form if the $\xi^{0}$ and $\xi^{\prime 0}$ are integrated out, where the $\hat{\mu}$-term (the 3-3-element in the inverse seesaw mass matrix) is suppressed by $m_{\xi}$.

Choosing the masses of the mediator fields at the $\mathrm{TeV}$ scale implies that the additional couplings are $\mathcal{O}\left(10^{-3}\right)$, which is comparable to the SM Yukawa couplings. Accordingly, this model can be tested at the LHC. The SM singlet fields $N$ and $N^{\prime}$ are only produced in small amounts due to the smallness of the Yukawa couplings. The SU(2) doublets $\xi$ and $\xi^{\prime}$, however, can be produced in Drell-Yan processes, similarly to charginos and neutralinos, with a cross-section of up to $\mathcal{O}\left(10^{2} \mathrm{fb}\right)$. These particles will then decay into vector bosons and leptons which is induced by the mixing between the neutral fermions as described by the mass matrix in Eq. (6). The smallness of the mixing between the heavy and the light neutrinos implies that one has to expect sizable decay length of up to several millimeters. Under favorable conditions one can even establish connections between neutrino physics and lepton number violating LHC signals arising from the production of two same sign charged leptons and several $W$-bosons.

The presence of the $\xi$ and $\xi^{\prime}$ supermultiplets modifies the running of the gauge couplings such that unification would not occur anymore. Therefore, one has to embed them into complete representations of a GUT gauge group. ${ }^{2}$ In the following we will focus on gauge groups that contain $\mathrm{SU}(5)$ as a subgroup. As the doublet mediators carry the same quantum numbers as the MSSM Higgs bosons the natural choices are $5_{\xi}$ and $\overline{5}_{\xi^{\prime}}$. The minimal field content is given by

$$
\overline{5}_{M}=\left(\begin{array}{c}
\left(d_{R}\right)^{c} \\
L
\end{array}\right), \quad \overline{5}_{\xi^{\prime}}=\left(\begin{array}{c}
d^{\prime c} \\
\xi^{\prime}
\end{array}\right), \quad 5_{\xi}=\left(\begin{array}{c}
d^{\prime \prime} \\
\xi
\end{array}\right), \quad H_{5}=\left(\begin{array}{c}
H_{\mathrm{col}} \\
H_{u}
\end{array}\right), \quad H_{\overline{5}}=\left(\begin{array}{c}
H_{\mathrm{col}}^{\prime} \\
H_{d}
\end{array}\right),
$$

and the fermionic singlets $N$ and $N^{\prime}$. This implies that at the electroweak scale one has not only the new leptonic fields $\xi^{\prime}$ and $\xi$, but also additional heavy quarks $d^{\prime c}$ and $d^{\prime \prime}$ stemming from $\overline{5}_{\xi^{\prime}}$ and $5_{\xi}$, respectively. We will discuss their phenomenology in section 4 . For completeness we recall that the fields $N$ and $N^{\prime}$ are (fermionic) gauge singlets, similar to the NMSSM singlet scalar field $S$. Note that in SUSY SU(5) models one usually needs additional Higgs 42-plets in order to describe the quark masses correctly. These 42-plets, however, are not relevant for the following discussion.

The most general $S U(5)$ invariant superpotential containing the MSSM fields, $\overline{5}_{\xi^{\prime}}, 5_{\xi}, N$ and $N^{\prime}$ reads as

$$
\begin{aligned}
W= & y_{1} N 5_{\xi} H_{\overline{5}}+y_{2} N \overline{5}_{\xi^{\prime}} H_{5}+y_{3} N \overline{5}_{M} H_{5}+ \\
& y_{1}^{\prime}, N^{\prime} 5_{\xi} H_{\overline{5}}+y_{2}^{\prime} N^{\prime} \overline{5}_{\xi^{\prime}} H_{5}+y_{3}^{\prime} N^{\prime} \overline{5}_{M} H_{5}+ \\
& m_{\xi^{\prime}} \overline{5}_{M} 5_{\xi}+m_{\xi} \overline{5}_{\xi^{\prime}} 5_{\xi}+m_{N} N^{\prime} N+m_{N N} N N+m_{N^{\prime} N^{\prime}} N^{\prime} N^{\prime}+ \\
& y_{d} \overline{5}_{M} 10 H_{\overline{5}}+y_{d}^{\prime} \overline{5}_{\xi^{\prime}} 10 H_{\overline{5}}+y_{u} 1010 H_{5}-\mu H_{\overline{5}} H_{5} .
\end{aligned}
$$

\footnotetext{
${ }^{2}$ In certain models it is possible to conserve gauge coupling unification without having complete multiplets of the GUT gauge group [? ]. The introduction of additional fields, however, is also required in these cases.
} 
Table 1: Possible $\mathbb{Z}_{3}$ assignments to forbid the Weinberg operator.

\begin{tabular}{c||cccccccc} 
Multiplet & $\overline{5}_{M}$ & $H_{5}$ & $H_{\overline{5}}$ & $N$ & $N^{\prime}$ & $5_{\xi}$ & $\overline{5}_{\xi^{\prime}}$ & 10 \\
\hline $\mathbb{Z}_{3}$ charge & 1 & 1 & 1 & 1 & 2 & 0 & 0 & 1
\end{tabular}

If both $y_{3}$ and $y_{3}^{\prime}$ were present, then one would generate the Weinberg operator which in general would be the dominant source for neutrino masses. However, if there were a discrete flavor symmetry operating at the GUT scale, then this operator could be forbidden, implying that the $d=7$ operator in Eq. (4) is the dominant contribution to neutrino mass. As an explicit example we have taken a $\mathbb{Z}_{3}$ symmetry with the charge assignments given in table 1 . The superpotential hence is then reduced to

$$
\begin{aligned}
W= & y_{3} N \overline{5}_{M} H_{5}+y_{1}^{\prime}, N^{\prime} 5_{\xi} H_{\overline{5}}+y_{2}^{\prime} N^{\prime} \overline{5}_{\xi^{\prime}} H_{5}+ \\
& m_{\xi} \overline{5}_{\xi^{\prime}} 5_{\xi}+m_{N} N^{\prime} N \\
& y_{d} \overline{5}_{M} 10 H_{\overline{5}}+y_{u} 1010 H_{5}-\mu H_{\overline{5}} H_{5} .
\end{aligned}
$$

The superpotential in Eq. (5) is obtained identifying $y_{3} \widehat{=} Y_{N}, y_{1}^{\prime} \widehat{=} \kappa_{1}$ and $y_{2}^{\prime} \hat{=} \kappa_{2}$. Note that the term $\mu H_{\overline{5}} H_{5}$ breaks the discrete symmetry explicitly (see discussion in Refs. [65, 71]). Furthermore, a somewhat problematic feature of this approach is, that the $\mu$-problem of the MSSM is enlarged as not only $\mu$, but also $m_{\xi}$ and $m_{N}$ have to be few hundred GeV up to at most a few $\mathrm{TeV}$.

Usually, one argues that the $\mu$ problem can be evaded if one enlarges the MSSM Higgs sector by a singlet $S$. This leads to the NMSSM, where $\mu=\lambda\left\langle v_{S}\right\rangle$ and $\lambda$ is the coupling between the MSSM Higgs doublets and $S$. Indeed, one could in principle also obtain $m_{\xi}$ and $m_{N}$ in this way in the desired order of magnitude. However, as we will discuss in the next section, this contradicts the requirement that the Weinberg operator should be forbidden.

\section{Implementation in the NMSSM, and the origin of a non-zero $\theta_{13}$ ?}

In the NMSSM, one introduces an extra gauge singlet $S$ which couples to the Higgs doublets such that $\mu=\lambda\langle S\rangle$. Similarly one can generate in this way also the mass terms for additional particles in the model discussed above. For this, the generalization of the superpotential in Eq. (9) reads as

$$
\begin{aligned}
W= & y_{1} N 5_{\xi} H_{\overline{5}}+y_{2} N \overline{5}_{\xi^{\prime}} H_{5}+y_{3} N \overline{5}_{M} H_{5}+ \\
& y_{1}^{\prime}, N^{\prime} 5_{\xi} H_{\overline{5}}+y_{2}^{\prime} N^{\prime} \overline{5}_{\xi^{\prime}} H_{5}+y_{3}^{\prime} N^{\prime} \overline{5}_{M} H_{5}+ \\
& \lambda_{\xi^{\prime}} S \overline{5}_{M} 5_{\xi}+\lambda_{\xi} S \overline{5}_{\xi^{\prime}} 5_{\xi}+\lambda_{N} S N^{\prime} N+\lambda_{N N} S N N+\lambda_{N^{\prime} N^{\prime}} S N^{\prime} N^{\prime}+ \\
& y_{d} \overline{5}_{M} 10 H_{\overline{5}}+y_{d}^{\prime} \overline{5}_{\xi^{\prime}} 10 H_{\overline{5}}+y_{u} 1010 H_{5} .
\end{aligned}
$$

After electroweak symmetry breaking, the heavy mass scale is automatically set by $\langle S\rangle$, which is of order of $\mathrm{TeV}$. This has immediate consequences for the effective neutrino mass operators, which have now the schematic structure

$$
\frac{1}{\langle S\rangle} L L H_{u} H_{u}, \quad \frac{1}{\langle S\rangle^{3}}\left(L L H_{u} H_{d}\right)\left(H_{u} H_{d}\right) .
$$

As a consequence $\langle S\rangle$ breaks any discrete symmetry under which it is charged. This prevents one from choosing a simple discrete symmetry group to avoid the $d=5$ contribution to neutrino mass. Especially the term $\lambda_{\xi^{\prime}} S \overline{5}_{M} 5_{\xi}$, which leads to a mixing between the light and heavy mediators is problematic in this context, as it induces the Weinberg operator.

We will prove now that one cannot forbid this term by a discrete symmetry that fulfills the required conditions. For this we start with three (yet) unconstrained charges as parameters

$$
q_{S} \equiv s, \quad q_{H_{5}} \equiv 2 h^{\prime}, \quad q_{N}=n .
$$




\begin{tabular}{c||cccccccccc} 
Multiplet & $\overline{5}_{M}$ & $H_{5}$ & $H_{\overline{5}}$ & $N$ & $N^{\prime}$ & $5_{\xi}$ & $\overline{5}_{\xi^{\prime}}$ & 10 & $S$ & $S^{\prime}$ \\
\hline $\mathbb{Z}_{3}$ charge & 1 & 1 & 1 & 1 & 2 & 0 & 0 & 1 & 1 & 0
\end{tabular}

Table 2: Charges for the fields of the model defined in Eq. (21).

From the absolutely necessary terms in the superpotential we derive

$\begin{array}{lll}\left(S H_{5} H_{\overline{5}}\right) & \Rightarrow & q_{H_{\overline{5}}}=-s-2 h^{\prime} \\ \left(S N N^{\prime}\right) & \Rightarrow & q_{N^{\prime}}=-s-n \\ \left(N \overline{5}_{M} H_{5}\right) & \Rightarrow & q_{M}=-2 h^{\prime}-n \\ \left(\overline{5}_{M} 10 \bar{H}_{\overline{5}}\right) & \Rightarrow & q_{10}=4 h^{\prime}+n+s .\end{array}$

From the term $\left(1010 H_{5}\right)$ we obtain

$$
n=-s-5 h^{\prime} .
$$

which leads to the following set of equations

$$
q_{H_{\overline{5}}}=-s-2 h^{\prime}, q_{N^{\prime}}=5 h^{\prime}, q_{M}=3 h^{\prime}+s, q_{10}=-h^{\prime} .
$$

As a consequence we derive for the charges of the doublets

$$
\begin{array}{lll}
\left(N^{\prime} H_{5} 5_{\xi}\right) & \Rightarrow & q_{\xi}=-3 h^{\prime}+s \\
\left(N^{\prime} \overline{5}_{\xi^{\prime}} H_{5}\right) & \Rightarrow & q_{\xi^{\prime}}=-7 h^{\prime}
\end{array}
$$

leading to

$$
q\left(S \overline{5}_{M} 5_{\xi}\right)=3 s,
$$

but we know that $3 s=0$ since we need a term $S^{3}$ in the NMSSM. This implies that one cannot forbid this unwanted term. Note, that this holds for every Abelian discrete symmetry group. As a consequence one can show along the same lines that every higher dimension operator for neutrino masses

$$
\mathcal{O}^{d}=\frac{1}{\langle S\rangle^{1+2 k+l}}\left(L L H_{u} H_{u}\right)\left(H_{u} H_{d}\right)^{k} S^{l}
$$

has to have the same charge as the Weinberg operator. We have also checked that similar problems appear if the Abelian symmetry is chosen as a product of two different cyclic groups $Z_{N} \otimes Z_{N^{\prime}}$.

The next possibility is the addition of a second singlet $S^{\prime}$ but this does not improve the situation either as one has to have interactions such that also $S^{\prime}$ obtains a VEV. However, from the term $S S S^{\prime}$ we obtain immediately $q_{S^{\prime}}=q_{S}$. As a consequence we find that the symmetry must be broken, which implies that also the Weinberg operator can be induced. However, if this breaking is small enough, then the resulting Weinberg operator contributes only sub-dominantly to the neutrino masses and mixing angles.

We consider the above model extended by a $S^{\prime}$ to exemplify the main points. The charges of the particles for the case of an unbroken symmetry are given in table 2 and the superpotential ${ }^{3}$ at the $S U(5)$ level is given by

$$
\begin{aligned}
W= & y_{3} N \overline{5}_{M} H_{5}+y_{1}^{\prime}, N^{\prime} 5_{\xi} H_{\overline{5}}+y_{2}^{\prime} N^{\prime} \overline{5}_{\xi^{\prime}} H_{5}+\lambda_{\xi} S^{\prime} \overline{5}_{\xi^{\prime}} 5_{\xi}+\lambda_{N} S^{\prime} N^{\prime} N \\
& +y_{d} \overline{5}_{M} 10 H_{\overline{5}}+y_{u} 1010 H_{5}+\lambda_{S} S H_{\overline{5}} H_{5}+\kappa S^{3}+\kappa^{\prime} S^{\prime 3} \\
& +\lambda_{S}^{\prime} S^{\prime} H_{\overline{5}} H_{5}+y_{3}^{\prime} N^{\prime} \overline{5}_{M} H_{5}+y_{d}^{\prime} \overline{5}_{\xi} 10 H_{\overline{5}}+\cdots,
\end{aligned}
$$

\footnotetext{
${ }^{3}$ Note that this charge assignment would allow for a quadratic mass term for $S^{\prime}$. However, we only allow for trilinear terms, which can be easily achieved, e.g., by adding an additional $\mathbb{Z}_{3}$ under which all fields have the same charge.
} 
where the interactions in the first two lines respect the discrete symmetry and the ones of the last line break it. The $\lambda_{S}^{\prime} S^{\prime} H_{\overline{5}} H_{5}$ term is a possibility to obtain a VEV for $S^{\prime}$ such that the mediators for the neutrino mass operators have their masses proportional to $\left\langle S^{\prime}\right\rangle$. The second term of this line yields the Weinberg operator after integrating out the heavy fields:

$$
m_{\nu}^{d=5}=\frac{y_{3} y_{3}^{\prime} v_{u}^{2}}{\lambda_{N}\left\langle S^{\prime}\right\rangle}
$$

We obtain from the requirement

$$
m_{\nu}^{d=5} \ll m_{\nu}^{d=7}=\frac{y_{1}^{\prime} y_{2}^{\prime} y_{3}^{2} v_{u}^{3} v_{d}}{\lambda_{N}^{2} \lambda_{\xi}\left\langle S^{\prime}\right\rangle^{3}}
$$

that $y_{3}^{\prime}<10^{-8}$ assuming (symmetry conserving) couplings of the order $10^{-2}$ and $\left\langle S^{\prime}\right\rangle \sim \mathrm{TeV}$. Hence we require all symmetry breaking couplings to be of order $10^{-8}$ or smaller. We have checked that $\lambda_{i}\left\langle S^{\prime}\right\rangle$ is of $O(\mathrm{TeV})$ in spite of $\lambda_{S}^{\prime}$ being so small.

It is interesting to observe that, for the symmetry breaking coupling $y_{3}^{\prime}$ close to this upper limit, the neutrino mass matrix in Eq. (23) could receive a new (and potentially large) contribution from the $d=5$ operator in Eq. (22). Suppose that the $d=7$ operator has a tri-bimaximal flavor structure, where (for the sake of simplicity) the charged lepton mass matrix is assumed to be diagonal. Then the deviation from tri-bimaximal mixing can originate in the symmetry breaking terms in Eq. (21). We illustrate this effect with an exemplary flavor structure, not discussing the details of the underlying flavor symmetry. For reason of simplicity, we use a minimal scenario where the mass of the lightest neutrino is zero. For the $d=7$ contribution, we assume a flavor structure where we have three generations of the singlet fields $N$ and $N^{\prime}$ and the following structure of the coupling constants ${ }^{4}$

$$
y_{1}^{\prime}=\widetilde{y}_{1}^{\prime}\left(\begin{array}{l}
0 \\
1 \\
\rho
\end{array}\right), \quad y_{2}^{\prime}=\widetilde{y}_{2}^{\prime}\left(\begin{array}{c}
0 \\
-1 \\
\rho
\end{array}\right), \quad y_{3}=\widetilde{y}_{3}\left(\begin{array}{ccc}
\sqrt{2 / 3} & 1 / \sqrt{3} & 0 \\
-1 / \sqrt{6} & 1 / \sqrt{3} & -1 / \sqrt{2} \\
-1 / \sqrt{6} & 1 / \sqrt{3} & 1 / \sqrt{2}
\end{array}\right),
$$

where $\widetilde{y}_{1}, \widetilde{y}_{2}$ and $\widetilde{y}_{3}$ are numerical (scalar) parameters, $\rho=\sqrt{m_{3} / m_{2}}$ and the mass matrices for $N$ and $N^{\prime}$ are diagonal. This choice of parameters will generate a tri-bimaximal mass matrix for the neutrinos at $d=7$

$$
\begin{aligned}
m_{\nu}^{d=7} & =\frac{v_{u}^{3} v_{d}}{\langle S\rangle^{3}} \cdot y_{3}\left[y_{1}^{\prime}\left(y_{2}^{\prime}\right)^{\top}+y_{2}^{\prime}\left(y_{1}^{\prime}\right)^{\top}\right] y_{3}^{\top} \\
& =U_{\mathrm{TB}} \cdot \operatorname{diag}\left(0, m_{2},-m_{3}\right) \cdot U_{\mathrm{TB}}^{\top}
\end{aligned}
$$

where $U_{\mathrm{TB}}$ is the tri-bimaximal mixing matrix. We can write the total neutrino mixing matrix including small linear deviations from tri-bimaximal mixing by using the parametrization from Ref. [89] (note the different sign convention)

$$
U_{\mathrm{PMNS}}=\left(\begin{array}{ccc}
\sqrt{\frac{2}{3}}\left(1-\frac{1}{2} s\right) & \frac{1}{\sqrt{3}}(1+s) & \frac{1}{\sqrt{2}} r \\
-\frac{1}{\sqrt{6}}(1+s-a-r) & \frac{1}{\sqrt{3}}\left(1-\frac{1}{2} s-a+r\right) & -\frac{1}{2}(1+a) \\
-\frac{1}{\sqrt{6}}(1+s+a+r) & \frac{1}{\sqrt{3}}\left(1-\frac{1}{2} s+a-r\right) & \frac{1}{2}(1-a)
\end{array}\right),
$$

where $\sin \theta_{13}=r / \sqrt{2}, \sin \theta_{12}=1 / \sqrt{3}(1+s)$, and $\sin \theta_{23}=1 / \sqrt{2}(1+a)$. Since we want to generate the corrections to the neutrino mass matrix by the $d=5$ contribution, we can write:

$$
\begin{gathered}
m_{\nu}=m_{\nu}^{d=7}+m_{\nu}^{d=5}, \quad \text { or } \\
U_{\mathrm{PMNS}} \cdot \operatorname{diag}\left(0, m_{2},-m_{3}\right) \cdot U_{\mathrm{PMNS}}^{\top}=U_{\mathrm{TB}} \cdot \operatorname{diag}\left(0, m_{2},-m_{3}\right) \cdot U_{\mathrm{TB}}^{\top}+\frac{v_{u}^{2}}{\langle S\rangle}\left(y_{3}\left(y_{3}^{\prime}\right)^{\top}+y_{3}^{\prime}\left(y_{3}\right)^{\top}\right)
\end{gathered}
$$

\footnotetext{
${ }^{4}$ Here we assume a similar flavor structure as in Ref. [71] but three instead of two generations of $N$ and $N^{\prime}$.
} 
Solving this equation for the symmetry breaking coupling $y_{3}^{\prime}$, we obtain

$$
y_{3}^{\prime} \simeq \widetilde{y}_{3}^{\prime}\left(\begin{array}{ccc}
\frac{s}{\sqrt{6}} & \frac{s}{\sqrt{3}} & \frac{(2 a-r)-3 r \rho^{2}}{3 \sqrt{2}} \\
\frac{s+(a+r) \rho^{2}}{\sqrt{6}} & \frac{-(2 a+r-s)-(2 a-r) \rho^{2}}{2 \sqrt{3}} & \frac{(2 a-r)+3 a \rho^{2}}{3 \sqrt{2}} \\
\frac{s-(a+r) \rho^{2}}{\sqrt{6}} & \frac{(2 a-r-s)+(2 a-r) \rho^{2}}{2 \sqrt{3}} & \frac{(2 a-r)+3 a \rho^{2}}{3 \sqrt{2}}
\end{array}\right)
$$

to first order in $r, s$, and $a$. If we want to consider only corrections to $\theta_{13}$ (i.e., $a=0, s=0$ ), we have,

$$
y_{3}^{\prime} \simeq \widetilde{y}_{3}^{\prime} r\left[\left(\begin{array}{ccc}
0 & 0 & -\frac{1}{\sqrt{2}} \\
\frac{1}{\sqrt{6}} & \frac{1}{2 \sqrt{3}} & 0 \\
-\frac{1}{\sqrt{6}} & -\frac{1}{2 \sqrt{3}} & 0
\end{array}\right) \rho^{2}-\left(\begin{array}{ccc}
0 & 0 & \frac{1}{3 \sqrt{2}} \\
0 & \frac{1}{2 \sqrt{3}} & \frac{1}{3 \sqrt{2}} \\
0 & \frac{1}{2 \sqrt{3}} & \frac{1}{3 \sqrt{2}}
\end{array}\right)\right]
$$

with $\mathcal{O}\left(\widetilde{y}_{3}^{\prime}\right)=10^{-8}$. This will give a correction to the tri-bimaximal mass matrix from the $d=7$ operator that leads to the observed neutrino mixing with $\theta_{13}>0$. In a specific model, such a structure may originate from a flavor symmetry.

\section{Phenomenology of the additional $d$-quarks}

The promotion of the additional leptonic SU(2) doublets $\xi$ and $\xi^{\prime}$ to 5 -plets $5_{\xi}$ and $\overline{5}_{\xi^{\prime}}$ of SU(5) implies the existence of additional $d$-quarks at the $\mathrm{TeV}$ scale as one wants to maintain gauge coupling unification. We will denote the corresponding mass eigenstates by $D^{\prime}$, which is composed of $d^{c \prime}$ and $d^{\prime \prime}$, and $L^{\prime}$ which is composed of $\xi$ and $\xi^{\prime}$. At the GUT-scale $L^{\prime}$ and $D^{\prime}$ have a common mass $m_{\xi, G U T}$. Renormalization group effects will lead to shift of the $L^{\prime}$ mass $m_{\xi}$ and the $D^{\prime}$ mass $m_{D^{\prime}}$. At one-loop order the corresponding RGEs read as

$$
\frac{d}{d t} m_{k}=\sum_{i} \frac{c_{i}^{k} \alpha_{i}(t)}{4 \pi} m_{k} \quad\left(k=\xi, D^{\prime}\right)
$$

where the index $i$ runs over the different gauge groups, $t=\ln \left(Q^{2} / M_{G U T}^{2}\right), c_{i}^{D^{\prime}}=(-4 / 15,0,-16 / 3)$ and $c_{i}^{\xi}=(-3 / 5,-3,0)$ for $i=U(1)_{Y}, S U(2)_{L}, S U(3)_{C}$. Here we have assumed that one can neglect the Yukawa interactions compared to the gauge interactions. These equations can easily be solved and one obtains

$$
m_{k}(t)=\prod_{i}\left(\frac{\alpha_{i}(t)}{\alpha_{G U T}}\right)^{\frac{c_{i}^{k}}{b_{i}}} m_{\xi, G U T}
$$

with $b_{i}=(38 / 5,2,-2)$. Clearly the precise ratio of these two masses at the electroweak scale depends on the particle content between the electroweak scale and the GUT scale as this changes the $b_{i}$. In our model we obtain $m_{D^{\prime}} / m_{\xi} \simeq 5$ at $Q=1 \mathrm{TeV}$. Provided that these leptons can be observed at LHC up to mass of about $800 \mathrm{GeV}[71,90]$ the $D^{\prime}$ can have a mass of up to $4 \mathrm{TeV}$ whereas LHC might observe them up to a mass of about $3 \mathrm{TeV}$ see e.g. [90] and Refs. therein.

Even though these quarks are heavier than the additional leptons, they will be stable in the model discussed so far as these particles are protected by the same symmetry that forbids the $d=5$ operator. Stable heavy d-quarks, however, cause conflicts with cosmological constraints. Their presence during Big Bang Nucleosynthesis (BBN) would alter the observed abundances of the light elements in the universe (see, e.g., Ref. [91] for a review). Further bounds come, e.g., from direct heavy element searches in water [92]. Although these additional quarks can annihilate via gluons into SM quarks and gluons, it turns out this mechanism is not sufficient to lower the yield below the experimental requirements [93, 94] if their masses are below $2.5 \mathrm{TeV}$, e.g. the range interesting for LHC. To summarize, heavy stable dquarks must have life-times much smaller than the age of the universe to avoid constraints from direct 
searches, and annihilation processes that are efficient enough to lower their abundance below the bounds from BBN. These bounds do not affect particles that decay before BBN $(\tau \ll 1 \mathrm{~s})$.

In order to let the $D^{\prime}$ decay fast enough, one therefore needs additional small terms that break the symmetry. For example the interactions of Eq. (21), discussed in the context of the NMSSM, lead to the two-body decays

$$
D^{\prime} \rightarrow H^{-} u, H^{0} d
$$

which result in life-times as small as $10^{-10} \mathrm{~s}$ to $10^{-13} \mathrm{~s}$, depending on $m_{D^{\prime}}$, if the symmetry breaking couplings are of order $10^{-8}$. The signal for such a small life-time at the LHC is a displaced vertex. Measuring the corresponding decay length immediately gives the size of the corresponding coupling.

\section{Systematic review of the decompositions, and their SU(5) comple- tions}

In the previous sections, we have discussed a specific realization of a supersymmetric model where the dominant contribution to neutrino masses is given by a $d=7$ operator. The complete list of $d=7$ operators including the various mediators is given in [71], where the previous model is listed as \#17. For convenience of the reader, we display here the possibilities in table 3 where we also include the $S U(5)$ multiplets containing the mediators.

The corresponding $S U(5)$ multiplets are either gauge singlets, 5-plets, 15-plets, 24-plets or 40-plets. The corresponding particles of the complete multiplet have to have masses at the $\mathrm{TeV}$ scale as not to destroy gauge coupling unification but only modifying the running of the gauge couplings. Using for example Ref. [96] one can easily calculate the additional contributions of these multiplets to the beta functions which we have summarized in table 4 . These values have to be compared with the MSSM beta functions $b_{i}=(33 / 5,1,-3)$ for $U(1)_{Y}, S U(2)_{L}$ and $S U(3)_{C}$, respectively. One sees that all but the 5 -plets give rather large contributions and one might wonder if such large contributions are consistent with the requirement of perturbativity up to the GUT scale.

At the one-loop level the RGE of the gauge coupling have the well-known solution

$$
\alpha_{G}=\frac{\alpha_{i}(Q)}{1-\frac{b_{i}}{4 \pi} \alpha_{i}(Q) \ln \frac{M_{G}^{2}}{Q^{2}}},
$$

where the $Q$ is the scale at which the additional particles are included, $M_{G} \simeq 2 \cdot 10^{16} \mathrm{GeV}$ is the GUT scale and $\alpha_{G}$ the value of the gauge couplings at $M_{G}$. Taking only the MSSM one gets $\alpha_{G} \simeq 1 / 25$. Adding a contribution $\Delta b_{i}$ to $b_{i}$ in Eq. (34) we find $\Delta b_{i}=5$ already implies that $\alpha_{G}=1$, see also [97] for a related discussion. As the 5-plet and the 15-plet are complex representations, they have to come always paired with a $\overline{5}$ and $\overline{15}$, respectively, as otherwise one cannot put a gauge invariant mass term in the superpotential. Inspecting table 4 we thus conclude that we can add at most five $5, \overline{5}$ pairs or one 24 -plet if one is willing to accept $\alpha_{G}=1$. If one would require $\alpha_{G} \leq 1 / 2$ then one is restricted to at most four $5, \overline{5}$ pairs. In practice the number is smaller as two-loop effects increase the value of $\alpha_{G}$ [84]. As a consequence, from the possible operators in table 3 only operators \#1, \#9, \#13 and \#17 are consistent with perturbativity up to the GUT scale. We note for completeness that in case of operator \#9 one has to include a corresponding $\overline{5}$ as otherwise no mass term can be added to the MSSM superpotential. Also for operators \#9 and \#13 the discussion as for operator \#17 in the previous sections can be repeated resulting in essentially the same findings. Note that decompositions \#1, \#9, and \#13 contain a singlet 


\begin{tabular}{|c|c|c|c|}
\hline$\overline{z \#}$ & Operator & Mediators & SU(5) multiplets \\
\hline 1 & $\left(H_{u} \mathrm{i} \tau^{2} \overline{L^{c}}\right)\left(H_{u} \mathrm{i} \tau^{2} L\right)\left(H_{d} \mathrm{i} \tau^{2} H_{u}\right)$ & $\mathbf{1}_{0}^{R}, \mathbf{1}_{0}^{L}, \mathbf{1}_{0}^{s}$ & $1,1,1$ \\
\hline 2 & $\left(H_{u} \mathrm{i} \tau^{2} \vec{\tau} \overline{L^{c}}\right)\left(H_{u} \mathrm{i} \tau^{2} L\right)\left(H_{d} \mathrm{i} \tau^{2} \vec{\tau} H_{u}\right)$ & $\mathbf{3}_{0}^{R}, \mathbf{3}_{0}^{L} \mathbf{1}_{0}^{R}, \mathbf{1}_{0}^{L}, \mathbf{3}_{0}^{s}$ & $24,24,(1),(1), 24$ \\
\hline 3 & $\left(H_{u} \mathrm{i} \tau^{2} \vec{\tau} \overline{L^{c}}\right)\left(H_{u} \mathrm{i} \tau^{2} \vec{\tau} L\right)\left(H_{d} \mathrm{i} \tau^{2} H_{u}\right)$ & $\mathbf{3}_{0}^{R}, \mathbf{3}_{0}^{L}, \mathbf{1}_{0}^{s}$ & $24,24,1$ \\
\hline 4 & $\left(-\mathrm{i} \epsilon^{a b c}\right)\left(H_{u} \mathrm{i} \tau^{2} \tau^{a} \overline{L^{c}}\right)\left(H_{u} \mathrm{i} \tau^{2} \tau^{b} L\right)\left(H_{d} \mathrm{i} \tau^{2} \tau^{c} H_{u}\right)$ & $\mathbf{3}_{0}^{R}, \mathbf{3}_{0}^{L}, \mathbf{3}_{0}^{s}$ & $24,24,24$ \\
\hline 5 & $\left(\overline{L^{c}} \mathrm{i} \tau^{2} \vec{\tau} L\right)\left(H_{d} \mathrm{i} \tau^{2} H_{u}\right)\left(H_{u} \mathrm{i} \tau^{2} \vec{\tau} H_{u}\right)$ & $\mathbf{3}_{+1}^{s}, \mathbf{3}_{+1}^{s}, \mathbf{1}_{0}^{s}$ & $15,15,1$ \\
\hline 6 & $\left(-\mathrm{i} \epsilon_{a b c}\right)\left(\overline{L^{c}} \mathbf{i} \tau^{2} \tau_{a} L\right)\left(H_{d} \mathrm{i} \tau^{2} \tau_{b} H_{u}\right)\left(H_{u} \mathrm{i} \tau^{2} \tau_{c} H_{u}\right)$ & $\mathbf{3}_{+1}^{s}, \mathbf{3}_{+1}^{s}, \mathbf{3}_{0}^{s}$ & $15,15,24$ \\
\hline 7 & $\left(H_{u} \mathrm{i} \tau^{2} \overline{L^{c}}\right)\left(L \mathrm{i} \tau^{2} \vec{\tau} H_{d}\right)\left(H_{u} \mathrm{i} \tau^{2} \vec{\tau} H_{u}\right)$ & $\mathbf{1}_{0}^{R}, \mathbf{1}_{0}^{L}, \mathbf{3}_{-1}^{R}, \mathbf{3}_{-1}^{L}, \mathbf{3}_{+1}^{s}$ & $1,1,15, \overline{15}, 15$ \\
\hline 8 & $\left(-\mathrm{i} \epsilon^{a b c}\right)\left(H_{u} \mathrm{i} \tau^{2} \tau^{a} \overline{L^{c}}\right)\left(L \mathrm{i} \tau^{2} \tau^{b} H_{d}\right)\left(H_{u} \mathrm{i} \tau^{2} \tau^{c} H_{u}\right)$ & $\mathbf{3}_{0}^{R}, \mathbf{3}_{0}^{L}, \mathbf{3}_{-1}^{R}, \mathbf{3}_{-1}^{L}, \mathbf{3}_{+1}^{s}$ & $24,24,15,15,15$ \\
\hline 9 & $\left(H_{u} \mathrm{i} \tau^{2} \overline{L^{c}}\right)\left(\mathrm{i} \tau^{2} H_{u}\right)(L)\left(H_{d} \mathrm{i} \tau^{2} H_{u}\right)$ & $\mathbf{1}_{0}^{R}, \mathbf{1}_{0}^{L}, \mathbf{2}_{-1 / 2}^{R}, \mathbf{2}_{-1 / 2}^{L}, \mathbf{1}_{0}^{s}$ & $\mathbf{1}, \mathbf{1}, \mathbf{5}, \overline{\mathbf{5}}, \mathbf{1}$ \\
\hline 10 & $\left(H_{u} \mathrm{i} \tau^{2} \vec{\tau} \overline{L^{c}}\right)\left(\mathrm{i} \tau^{2} \vec{\tau} H_{u}\right)(L)\left(H_{d} \mathrm{i} \tau^{2} H_{u}\right)$ & $\mathbf{3}_{0}^{R}, \mathbf{3}_{0}^{L}, \mathbf{2}_{-1 / 2}^{R}, \mathbf{2}_{-1 / 2}^{L}, \mathbf{1}_{0}^{s}$ & $24,24,5, \overline{5}, 1$ \\
\hline 11 & $\left(H_{u} \mathrm{i} \tau^{2} \overline{L^{c}}\right)\left(\mathrm{i} \tau^{2} H_{u}\right)(\vec{\tau} L)\left(H_{d} \mathrm{i} \tau^{2} \vec{\tau} H_{u}\right)$ & $\mathbf{1}_{0}^{R}, \mathbf{1}_{0}^{L}, \mathbf{2}_{-1 / 2}^{R}, \mathbf{2}_{-1 / 2}^{L}, \mathbf{3}_{0}^{s}$ & $1,1,5, \overline{5}, 24$ \\
\hline 12 & $\left(H_{u} \mathrm{i} \tau^{2} \tau^{a} \overline{L^{c}}\right)\left(\mathrm{i} \tau^{2} \tau^{a} H_{u}\right)\left(\tau^{b} L\right)\left(H_{d} \mathrm{i} \tau^{2} \tau^{b} H_{u}\right)$ & $\mathbf{3}_{0}^{R}, \mathbf{3}_{0}^{L}, \mathbf{2}_{-1 / 2}^{R}, \mathbf{2}_{-1 / 2}^{L}, \mathbf{3}_{0}^{s}$ & $24,24,5, \overline{5}, 24$ \\
\hline 13 & $\left(H_{u} \mathrm{i} \tau^{2} \overline{L^{c}}\right)(L)\left(\mathrm{i} \tau^{2} H_{u}\right)\left(H_{d} \mathrm{i} \tau^{2} H_{u}\right)$ & $\mathbf{1}_{0}^{R}, \mathbf{1}_{0}^{L}, \mathbf{2}_{+1 / 2}^{s}, \mathbf{1}_{0}^{s}$ & $1,1,5,1$ \\
\hline 14 & $\left(H_{u} \mathrm{i} \tau^{2} \vec{\tau} \overline{L^{c}}\right)(\vec{\tau} L)\left(\mathrm{i} \tau^{2} H_{u}\right)\left(H_{d} \mathrm{i} \tau^{2} H_{u}\right)$ & $\mathbf{3}_{0}^{R}, \mathbf{3}_{0}^{L}, \mathbf{2}_{+1 / 2}^{s}, \mathbf{1}_{0}^{s}$ & $24,24,5,1$ \\
\hline 15 & $\left(H_{u} \mathrm{i} \tau^{2} \overline{L^{c}}\right)(L)\left(\mathrm{i} \tau^{2} \vec{\tau} H_{u}\right)\left(H_{d} \mathrm{i} \tau^{2} \vec{\tau} H_{u}\right)$ & $\mathbf{1}_{0}^{R}, \mathbf{1}_{0}^{L}, \mathbf{2}_{+1 / 2}^{s}, \mathbf{3}_{0}^{s}$ & $1,1,5,24$ \\
\hline 16 & $\left(H_{u} \mathrm{i} \tau^{2} \tau^{a} \overline{L^{c}}\right)\left(\tau^{a} L\right)\left(\mathrm{i} \tau^{2} \tau^{b} H_{u}\right)\left(H_{d} \mathrm{i} \tau^{2} \tau^{b} H_{u}\right)$ & $\mathbf{3}_{0}^{R}, \mathbf{3}_{0}^{L}, \mathbf{2}_{+1 / 2}^{s}, \mathbf{3}_{0}^{s}$ & $24,24,5,24$ \\
\hline 17 & $\left(H_{u} \mathrm{i} \tau^{2} \overline{L^{c}}\right)\left(H_{d}\right)\left(\mathrm{i} \tau^{2} H_{u}\right)\left(H_{u} \mathrm{i} \tau^{2} L\right)$ & $\mathbf{1}_{0}^{R}, \mathbf{1}_{0}^{L}, \mathbf{2}_{-1 / 2}^{R}, \mathbf{2}_{-1}^{L}$ & $1,1,5, \overline{5}$ \\
\hline 18 & $\left(H_{u} \mathrm{i} \tau^{2} \vec{\tau} \overline{L^{c}}\right)\left(\vec{\tau} H_{d}\right)\left(\mathrm{i} \tau^{2} H_{u}\right)\left(H_{u} \mathrm{i} \tau^{2} L\right)$ & $\mathbf{3}_{0}^{R}, \mathbf{3}_{0}^{L}, \mathbf{2}_{-1 / 2}^{R}, \mathbf{2}_{-1 / 2}^{L}, \mathbf{1}_{0}^{R}, \mathbf{1}_{0}^{L}$ & $24,24,5, \overline{5},(1),(1)$ \\
\hline 19 & $\left(H_{u} \mathrm{i} \tau^{2} \overline{L^{c}}\right)\left(H_{d}\right)\left(\mathrm{i} \tau^{2} \vec{\tau} H_{u}\right)\left(H_{u} \mathrm{i} \tau^{2} \vec{\tau} L\right)$ & $\mathbf{1}_{0}^{R}, \mathbf{1}_{0}^{L}, \mathbf{2}_{-1 / 2}^{R}, \mathbf{2}_{-1 / 2}^{L}, \mathbf{3}_{0}^{R}, \mathbf{3}_{0}^{L}$ & $(\mathbf{1}),(\mathbf{1}), 5, \overline{5}, 24,24$ \\
\hline 20 & $\left(H_{u} \mathrm{i} \tau^{2} \tau^{a} \overline{L^{c}}\right)\left(\tau^{a} H_{d}\right)\left(\mathrm{i} \tau^{2} \tau^{b} H_{u}\right)\left(H_{u} \mathrm{i} \tau^{2} \tau^{b} L\right)$ & $\mathbf{3}_{0}^{R}, \mathbf{3}_{0}^{L}, \mathbf{2}_{-1 / 2}^{R}, \mathbf{2}_{-1 / 2}^{L}$ & $24,24,5, \overline{5}$ \\
\hline 21 & $\left(\overline{L^{c}} \mathrm{i} \tau^{2} \tau^{a} L\right)\left(H_{u} \mathrm{i} \tau^{2} \tau^{a}\right)\left(\tau^{b} H_{d}\right)\left(H_{u} \mathrm{i} \tau^{2} \tau^{b} H_{u}\right)$ & $\mathbf{3}_{+1}^{s}, \mathbf{2}_{+1 / 2}^{s}, \mathbf{3}_{+1}^{s}$ & $15,5,15$ \\
\hline 22 & $\left(\overline{L^{c}} \mathbf{i} \tau^{2} \tau^{a} L\right)\left(H_{d} \mathrm{i} \tau^{2} \tau^{a}\right)\left(\tau^{b} H_{u}\right)\left(H_{u} \mathrm{i} \tau^{2} \tau^{b} H_{u}\right)$ & $\mathbf{3}_{+1}^{s}, \mathbf{2}_{+3 / 2}^{s}, \mathbf{3}_{+1}^{s}$ & $15,40,15$ \\
\hline 23 & $\left(\overline{L^{c}} \mathrm{i} \tau^{2} \vec{\tau} L\right)\left(H_{u} \mathrm{i} \tau^{2} \vec{\tau}\right)\left(H_{u}\right)\left(H_{d} \mathrm{i} \tau^{2} H_{u}\right)$ & $\mathbf{3}_{+1}^{s}, \mathbf{2}_{+1 / 2}^{s}, \mathbf{1}_{0}^{s}$ & $15,5,1$ \\
\hline 24 & $\left(\overline{L^{c}} \mathbf{i} \tau^{2} \tau^{a} L\right)\left(H_{u} \mathrm{i} \tau^{2} \tau^{a}\right)\left(\tau^{b} H_{u}\right)\left(H_{d} \mathrm{i} \tau^{2} \tau^{b} H_{u}\right)$ & $\mathbf{3}_{+1}^{s}, \mathbf{2}_{+1 / 2}^{s}, \mathbf{3}_{0}^{s}$ & $15,5,24$ \\
\hline 25 & $\left(H_{d} \mathrm{i} \tau^{2} H_{u}\right)\left(\overline{L^{c}} \mathrm{i} \tau^{2}\right)(\vec{\tau} L)\left(H_{u} \mathrm{i} \tau^{2} \vec{\tau} H_{u}\right)$ & $\mathbf{1}_{0}^{s}, \mathbf{2}_{+1 / 2}^{L}, \mathbf{2}_{+1 / 2}^{R}, \mathbf{3}_{+1}^{s}$ & $1,5, \overline{5}, 15$ \\
\hline 26 & $\left(H_{d} \mathrm{i} \tau^{2} \tau^{a} H_{u}\right)\left(\overline{L^{c}} \mathrm{i} \tau^{2} \tau^{a}\right)\left(\tau^{b} L\right)\left(H_{u} \mathrm{i} \tau^{2} \tau^{b} H_{u}\right)$ & $\mathbf{3}_{0}^{s}, \mathbf{2}_{+1 / 2}^{L}, \mathbf{2}_{+1 / 2}^{R}, \mathbf{3}_{+1}^{s}$ & $24,5, \overline{5}, 15$ \\
\hline 27 & $\left(H_{u} \mathrm{i} \tau^{2} \bar{L}^{c}\right)\left(\mathrm{i} \tau^{2} H_{d}\right)(\vec{\tau} L)\left(H_{u} \mathrm{i} \tau^{2} \vec{\tau} H_{u}\right)$ & $\mathbf{1}_{0}^{R}, \mathbf{1}_{0}^{L}, \mathbf{2}_{+1 / 2}^{R}, \mathbf{2}_{+1 / 2}^{L}, \mathbf{3}_{+1}^{s}$ & $1,1,5, \overline{5}, 15$ \\
\hline 28 & $\left(H_{u} \mathrm{i} \tau^{2} \tau^{a} \overline{L^{c}}\right)\left(\mathrm{i} \tau^{2} \tau^{a} H_{d}\right)\left(\tau^{b} L\right)\left(H_{u} \mathrm{i} \tau^{2} \tau^{b} H_{u}\right)$ & $\mathbf{3}_{0}^{R}, \mathbf{3}_{0}^{L}, \mathbf{2}_{+1 / 2}^{R}, \mathbf{2}_{+1 / 2}^{L}, \mathbf{3}_{+1}^{s}$ & $24,24,5, \overline{5}, 15$ \\
\hline 29 & $\left(H_{u} \mathrm{i} \tau^{2} \overline{L^{c}}\right)(L)\left(\mathrm{i} \tau^{2} \vec{\tau} H_{d}\right)\left(H_{u} \mathrm{i} \tau^{2} \vec{\tau} H_{u}\right)$ & $\mathbf{1}_{0}^{R}, \mathbf{1}_{0}^{L}, \mathbf{2}_{+1 / 2}^{s}, \mathbf{3}_{+1}^{s}$ & $1,1,5,15$ \\
\hline 30 & $\left(H_{u} \mathrm{i} \tau^{2} \tau^{a} \overline{L^{c}}\right)\left(\tau^{a} L\right)\left(\mathrm{i} \tau^{2} \tau^{b} H_{d}\right)\left(H_{u} \mathrm{i} \tau^{2} \tau^{b} H_{u}\right)$ & $\mathbf{3}_{0}^{R}, \mathbf{3}_{0}^{L}, \mathbf{2}_{+1 / 2}^{s}, \mathbf{3}_{+1}^{s}$ & $24,24,5,15$ \\
\hline 31 & $\left(\overline{L^{c}} \mathrm{i} \tau^{2} \tau^{a} H_{d}\right)\left(\mathrm{i} \tau^{2} \tau^{a} H_{u}\right)\left(\tau^{b} L\right)\left(H_{u} \mathrm{i} \tau^{2} \tau^{b} H_{u}\right)$ & $\mathbf{3}_{+1}^{L}, \mathbf{3}_{+1}^{R}, \mathbf{2}_{+1 / 2}^{L}, \mathbf{2}_{+1 / 2}^{R}, \mathbf{3}_{+1}^{s}$ & $15, \overline{15}, 5, \overline{5}, 15$ \\
\hline 32 & $\left(\overline{L^{c}} \mathrm{i} \tau^{2} \tau^{a} H_{d}\right)\left(\tau^{a} L\right)\left(\mathrm{i} \tau^{2} \tau^{b} H_{u}\right)\left(H_{u} \mathrm{i} \tau^{2} \tau^{b} H_{u}\right)$ & $\mathbf{3}_{+1}^{L}, \mathbf{3}_{+1}^{R}, \mathbf{2}_{+3 / 2}^{s}, \mathbf{3}_{+1}^{s}$ & $15, \overline{15}, 40,15$ \\
\hline 33 & $\left(\overline{L^{c}} \mathrm{i} \tau^{2} \vec{\tau} H_{d}\right)\left(\mathrm{i} \tau^{2} \vec{\tau} H_{u}\right)\left(H_{u}\right)\left(H_{u} \mathrm{i} \tau^{2} L\right)$ & $\mathbf{3}_{+1}^{L}, \mathbf{3}_{+1}^{R}, \mathbf{2}_{+1 / 2}^{L}, \mathbf{2}_{+1 / 2}^{R}, \mathbf{1}_{0}^{L}, \mathbf{1}_{0}^{R}$ & $15, \overline{15}, 5, \overline{5}, 1,1$ \\
\hline 34 & $\left(\overline{L^{c}} \mathrm{i} \tau^{2} \tau^{a} H_{d}\right)\left(\mathrm{i} \tau^{2} \tau^{a} H_{u}\right)\left(\tau^{b} H_{u}\right)\left(H_{u} \mathrm{i} \tau^{2} \tau^{b} L\right)$ & $\mathbf{3}_{+1}^{L}, \mathbf{3}_{+1}^{R}, \mathbf{2}_{+1 / 2}^{L}, \mathbf{2}_{+1 / 2}^{R}, \mathbf{3}_{0}^{L}, \mathbf{3}_{0}^{R}$ & $15, \overline{15}, 5, \overline{5}, 24,24$ \\
\hline
\end{tabular}

Table 3: Decompositions of the $d=7$ operator $L L H_{u} H_{u} H_{d} H_{u}$ at tree level. We use the following notation for the mediators: $\mathbf{X}_{\mathbf{Y}}^{\mathbf{L}}$. The $\mathrm{X}$ describes the $\mathrm{SU}(2)$ nature, i.e., singlet (1), doublet (2), or triplet (3). The superscript $L$ denotes a left- (L) or right- (R) handed fermion, or a scalar (s). The subscript $Y$ represents the hypercharge $Y \equiv Q-I_{3}^{W}$. The SU(5) singlets in parentheses can be already contained in the 24-plets. 


\begin{tabular}{c||cccc} 
Multiplet & 5 & 15 & 24 & 40 \\
\hline$\Delta b_{i}$ & $1 / 2$ & $7 / 2$ & 5 & 11
\end{tabular}

Table 4: Contributions of the various $S U(5)$ multiplets to the MSSM beta functions.

scalar in the decomposition. Looking at the according superpotentials

$$
\begin{gathered}
W_{\# 1}=\overline{5}_{M} 10 H_{\overline{5}}+1010 H_{5}+S S S+S H_{5} H_{\overline{5}}+H_{5} \overline{5}_{M} N+H_{5} H_{\overline{5}} \phi+ \\
N^{\prime} N^{\prime} \phi+N N^{\prime} S+\phi \phi S \\
W_{\# 3}=\overline{5}_{M} 10 H_{\overline{5}}+1010 H_{5}+S S S+S H_{5} H_{\overline{5}}+H_{5} \overline{5}_{M} 24_{a}+H_{5} H_{\overline{5}} \phi+ \\
24_{b} 24_{b} \phi+24_{a} 24_{b} S+\phi \phi S \\
W_{\# 13=} \overline{5}_{M} 10 H_{\overline{5}}+1010 H_{5}+S S S+S H_{5} H_{\overline{5}}+\overline{5}_{M} H_{5} N+N^{\prime} \overline{5}_{M} 5_{s}+ \\
\overline{5}_{s} H_{5} \phi+H_{5} H_{\overline{5}} \phi+N N^{\prime} S+\overline{5}_{s} 5_{s} S+\phi \phi S,
\end{gathered}
$$

where the $\phi$ are the scalar singlets and for the other fields we use the notation introduced in the previous sections. Note, that we only give the superfields but not the details of the couplings. All three superpotentials contain the term $H_{5} H_{\overline{5}} \phi$, which eventually induces a vev for the scalar component of $\phi$. In these cases, a lower than $d=7$ dimensional operator will dominate neutrino mass. This means that our example in Fig. 1 is the only tree level decomposition which is consistent with perturbativity up to the GUT scale and neutrino mass generation by a leading $d=7$ contribution.

\section{Summary and conclusions}

We have discussed neutrino mass generation from a $d=7$ effective operator, while the $d=5$ Weinberg operator is suppressed due to a discrete symmetry. The additional suppression by $v^{2} / \Lambda_{\mathrm{NP}}^{2}$ compared to the Weinberg operator reduces the new physics scale $\Lambda_{\mathrm{NP}}$ required to describe the smallness of neutrino mass, potentially down to the $\mathrm{TeV}$ scale, which makes the mediators accessible at the LHC. We have especially focused on TeV completions in the MSSM and NMSSM frameworks. In order to achieve gauge coupling unification, we have embedded such models in a GUT scenario with an SU(5) subgroup.

We have chosen one specific example for the decomposition of the $d=7$ operator in Eq. (4), see Fig. 1, which has been discussed earlier in the context of its LHC phenomenology [71]. It involves two fermionic $\mathrm{SU}(2)$ singlets, and two fermionic $\mathrm{SU}(2)$ doublets. In this case, not only the mediators may be probed at the LHC, but also sensitivity to lepton flavor and perhaps even lepton number violation can be achieved. In this study, we have demonstrated that this example is peculiar for two reasons: 1) it can be completed in an SU(5) and described perturbatively up to the GUT scale, and 2) it does not contain any singlet scalars which take a vev and thereby generate neutrino mass by a lower dimensional operator. It is, in fact, the only example which satisfies these two requirements.

The embedding of the fermionic doublets into SU(2) multiplets implies that additional heavy $d$ quarks are to be introduced, which are strongly constrained by early universe cosmology, such as big bang nucleosynthesis. Therefore, fast enough decay mechanisms for these $d$-quarks need to be identified. The decay via leptoquarks or higher dimensional operators, which may be generated from physics beyond the GUT scale, is possible, but nevertheless too slow to satisfy the current constraints. The possibility in agreement with cosmological bounds is a decay via a small (discrete) symmetry breaking term, which could be large enough to allow for a sufficient decay rate.

For the specific case of the NMSSM, we have demonstrated that the NMSSM scalar vev $\langle S\rangle$ breaks the discrete symmetry spontaneously, which will lead to a (dominating) neutrino mass term from the Weinberg operator. A simple way out has been shown to introduce an additional scalar and symmetry breaking terms, which can be small enough such that the $d=5$ contribution to neutrino mass is suppressed. We have also shown that these small symmetry breaking terms may describe the origin of deviations from tri-bimaximal mixing, and this may be the origin of the non-vanishing magnitude of $\theta_{13}$. 
In conclusion, we have discussed a model which described neutrino mass from physics at the $\mathrm{TeV}$ scale, and which is potentially LHC-testable. We have embedded the model in an SU(5) SUSY-GUT, which means that we have confirmed gauge coupling unification and perturbativity up to the GUT scale. Finally, we have established that this model requires the fast enough decay of heavy $d$-quarks, which are needed for the GUT completion of the theory. Therefore, interesting constraints will not only be obtained from LHC, but also from early universe cosmology.

\section{Acknowledgments}

M.B.K. likes to thank the physics department of Università Roma Tre for the hospitality during his stay in Rome. This work has been supported by the DFG research training group GRK 1147. W.P. has been supported by the DFG project number PO1337/2-1. W.W. would like to acknowledge support from DFG grants WI 2639/3-1 and WI 2639/4-1. This work has also been supported by the FP7 Invisibles network (Marie Curie Actions, PITN-GA-2011-289442). D.M. acknowledges MIUR (Italy) for financial support under the program "Futuro in Ricerca 2010 (RBFR10O36O)". 


\section{References}

[1] DAYA-BAY Collaboration, F. An et al., Phys.Rev.Lett. 108, 171803 (2012), arXiv:1203.1669.

[2] RENO collaboration, J. Ahn et al., Phys.Rev.Lett. 108, 191802 (2012), arXiv:1204.0626.

[3] G. Altarelli and F. Feruglio, Rev.Mod.Phys. 82, 2701 (2010), arXiv:1002.0211.

[4] ATLAS Collaboration, G. Aad et al., Phys.Lett. B716, 1 (2012), arXiv:1207.7214.

[5] CMS Collaboration, S. Chatrchyan et al., Phys.Lett. B716, 30 (2012), arXiv:1207.7235.

[6] ATLAS Collaboration, G. Aad et al., (2012), arXiv:1208.0949.

[7] CMS Collaboration, S. Chatrchyan et al., (2012), arXiv:1206.3949.

[8] ATLAS Collaboration, G. Aad et al., (2012), arXiv:1211.6312.

[9] CMS Collaboration, S. Chatrchyan et al., JHEP 1210, 018 (2012), arXiv:1207.1798.

[10] CMS Collaboration, S. Chatrchyan et al., Phys.Rev.Lett. 109, 171803 (2012), arXiv:1207.1898.

[11] ATLAS Collaboration, G. Aad et al., (2012), arXiv:1210.7451.

[12] ATLAS Collaboration, G. Aad et al., (2012), arXiv:1211.1597.

[13] CMS Collaboration, S. Chatrchyan et al., (2012), arXiv:1210.5629.

[14] CMS Collaboration, S. Chatrchyan et al., (2012), arXiv:1211.4784.

[15] ATLAS Collaboration, G. Aad et al., (2012), arXiv:1212.1272.

[16] P. Bechtle et al., JHEP 1206, 098 (2012), arXiv:1204.4199.

[17] O. Buchmueller et al., Eur.Phys.J. C72, 2243 (2012), arXiv:1207.7315.

[18] M. Papucci, J. T. Ruderman, and A. Weiler, JHEP 1209, 035 (2012), arXiv:1110.6926.

[19] R. Mahbubani, M. Papucci, G. Perez, J. T. Ruderman, and A. Weiler, (2012), arXiv:1212.3328.

[20] J. L. Feng and D. Sanford, Phys.Rev. D86, 055015 (2012), arXiv:1205.2372.

[21] H. Baer, V. Barger, P. Huang, and X. Tata, JHEP 1205, 109 (2012), arXiv:1203.5539.

[22] B. Allanach and B. Gripaios, JHEP 1205, 062 (2012), arXiv:1202.6616.

[23] Z. Han, A. Katz, M. Son, and B. Tweedie, (2012), arXiv:1211.4025.

[24] U. Ellwanger, JHEP 1203, 044 (2012), arXiv:1112.3548.

[25] J. F. Gunion, Y. Jiang, and S. Kraml, Phys.Rev. D86, 071702 (2012), arXiv:1207.1545.

[26] J. F. Gunion, Y. Jiang, and S. Kraml, Phys.Lett. B710, 454 (2012), arXiv:1201.0982.

[27] D. Das, U. Ellwanger, and A. M. Teixeira, JHEP 1204, 067 (2012), arXiv:1202.5244.

[28] G. G. Ross, K. Schmidt-Hoberg, and F. Staub, JHEP 1208, 074 (2012), arXiv:1205.1509.

[29] S. F. King, M. Mühlleitner, R. Nevzorov, and K. Walz, (2012), arXiv:1211.5074.

[30] H. E. Haber and M. Sher, Phys.Rev. D35, 2206 (1987).

[31] M. Drees, Phys.Rev. D35, 2910 (1987). 
[32] M. Cvetic, D. A. Demir, J. Espinosa, L. Everett, and P. Langacker, Phys.Rev. D56, 2861 (1997), arXiv:hep-ph/9703317.

[33] Y. Zhang, H. An, X.-d. Ji, and R. N. Mohapatra, Phys.Rev. D78, 011302 (2008), arXiv:0804.0268.

[34] M. Hirsch, M. Malinsky, W. Porod, L. Reichert, and F. Staub, JHEP 1202, 084 (2012), arXiv:1110.3037.

[35] B. O’Leary, W. Porod, and F. Staub, JHEP 1205, 042 (2012), arXiv:1112.4600.

[36] I. Gogoladze, F. Nasir, and Q. Shafi, (2012), arXiv:1212.2593.

[37] P. Minkowski, Phys. Lett. B67, 421 (1977).

[38] T. Yanagida, (1979), In Proceedings of the Workshop on the Baryon Number of the Universe and Unified Theories, Tsukuba, Japan, 13-14 Feb 1979.

[39] M. Gell-Mann, P. Ramond, and R. Slansky, (1979), Print-80-0576 (CERN).

[40] R. N. Mohapatra and G. Senjanovic, Phys. Rev. Lett. 44, 912 (1980).

[41] S. Weinberg, Phys. Rev. Lett. 43, 1566 (1979).

[42] E. Ma, Phys. Rev. Lett. 81, 1171 (1998), arXiv:hep-ph/9805219.

[43] K. Babu and C. N. Leung, Nucl.Phys. B619, 667 (2001), arXiv:hep-ph/0106054.

[44] A. de Gouvea and J. Jenkins, Phys.Rev. D77, 013008 (2008), arXiv:0708.1344.

[45] F. Bonnet, M. Hirsch, T. Ota, and W. Winter, JHEP 1207, 153 (2012), arXiv:1204.5862.

[46] Y. Farzan, S. Pascoli, and M. A. Schmidt, (2012), arXiv:1208.2732.

[47] P. W. Angel, N. L. Rodd, and R. R. Volkas, (2012), arXiv:1212.6111.

[48] R. Mohapatra and J. Valle, Phys. Rev. D34, 1642 (1986).

[49] R. Hempfling, Nucl.Phys. B478, 3 (1996), arXiv:hep-ph/9511288.

[50] H.-P. Nilles and N. Polonsky, Nucl.Phys. B484, 33 (1997), arXiv:hep-ph/9606388.

[51] F. Borzumati, Y. Grossman, E. Nardi, and Y. Nir, Phys.Lett. B384, 123 (1996), arXiv:hep$\mathrm{ph} / 9606251$.

[52] E. Nardi, Phys.Rev. D55, 5772 (1997), arXiv:hep-ph/9610540.

[53] E. Chun, S. Kang, C. Kim, and U. Lee, Nucl.Phys. B544, 89 (1999), arXiv:hep-ph/9807327.

[54] M. Hirsch, M. Diaz, W. Porod, J. Romão, and J. Valle, Phys.Rev. D62, 113008 (2000), arXiv:hep$\mathrm{ph} / 0004115$.

[55] M. Diaz, M. Hirsch, W. Porod, J. Romão, and J. Valle, Phys.Rev. D68, 013009 (2003), arXiv:hep$\mathrm{ph} / 0302021$.

[56] A. Dedes, S. Rimmer, and J. Rosiek, JHEP 0608, 005 (2006), arXiv:hep-ph/0603225.

[57] B. Allanach and C. Kom, JHEP 0804, 081 (2008), arXiv:0712.0852.

[58] P. Ghosh and S. Roy, JHEP 0904, 069 (2009), arXiv:0812.0084.

[59] K. S. Babu and S. Nandi, Phys. Rev. D62, 033002 (2000), arXiv:hep-ph/9907213. 
[60] M.-C. Chen, A. de Gouvea, and B. A. Dobrescu, Phys. Rev. D75, 055009 (2007), arXiv:hep$\mathrm{ph} / 0612017$.

[61] I. Gogoladze, N. Okada, and Q. Shafi, Phys. Lett. B672, 235 (2009), arXiv:0809.0703.

[62] G. F. Giudice and O. Lebedev, Phys. Lett. B665, 79 (2008), arXiv:0804.1753.

[63] K. S. Babu, S. Nandi, and Z. Tavartkiladze, Phys. Rev. D80, 071702 (2009), arXiv:0905.2710.

[64] P.-H. Gu, H.-J. He, U. Sarkar, and X.-m. Zhang, Phys. Rev. D80, 053004 (2009), arXiv:0906.0442.

[65] F. Bonnet, D. Hernandez, T. Ota, and W. Winter, JHEP 10, 076 (2009), arXiv:0907.3143.

[66] I. Picek and B. Radovcic, Phys. Lett. B687, 338 (2010), arXiv:0911.1374.

[67] Y. Liao, G.-Z. Ning, and L. Ren, Phys. Rev. D82, 113003 (2010), arXiv:1008.0117.

[68] Y. Liao, Phys. Lett. B694, 346 (2011), arXiv:1009.1692.

[69] Y. Liao, Phys. Lett. B698, 288 (2011), arXiv:1010.5326.

[70] S. Kanemura and T. Ota, Phys. Lett. B694, 233 (2010), arXiv:1009.3845.

[71] M. B. Krauss, T. Ota, W. Porod, and W. Winter, Phys.Rev. D84, 115023 (2011), arXiv:1109.4636.

[72] S. Dimopoulos, S. Raby, and F. Wilczek, Phys.Rev. D24, 1681 (1981).

[73] L. E. Ibanez and G. G. Ross, Phys.Lett. B105, 439 (1981).

[74] W. J. Marciano and G. Senjanovic, Phys.Rev. D25, 3092 (1982).

[75] M. Einhorn and D. Jones, Nucl.Phys. B196, 475 (1982).

[76] U. Amaldi, W. de Boer, and H. Fürstenau, Phys.Lett. B260, 447 (1991).

[77] P. Langacker and M.-x. Luo, Phys.Rev. D44, 817 (1991).

[78] J. R. Ellis, S. Kelley, and D. V. Nanopoulos, Phys.Lett. B260, 131 (1991).

[79] A. Rossi, Phys.Rev. D66, 075003 (2002), arXiv:hep-ph/0207006.

[80] M. R. Buckley and H. Murayama, Phys.Rev.Lett. 97, 231801 (2006), arXiv:hep-ph/0606088.

[81] M. Hirsch, S. Kaneko, and W. Porod, Phys.Rev. D78, 093004 (2008), arXiv:0806.3361.

[82] J. Esteves et al., JHEP 0905, 003 (2009), arXiv:0903.1408.

[83] F. Borzumati and T. Yamashita, Prog.Theor.Phys. 124, 761 (2010), arXiv:0903.2793.

[84] J. Esteves, J. Romão, M. Hirsch, F. Staub, and W. Porod, Phys.Rev. D83, 013003 (2011), arXiv:1010.6000.

[85] C. Biggio and L. Calibbi, JHEP 1010, 037 (2010), arXiv:1007.3750.

[86] C. Biggio, L. Calibbi, A. Masiero, and S. K. Vempati, JHEP 1208, 150 (2012), arXiv:1205.6817.

[87] M. Hirsch, W. Porod, F. Staub, and C. Weiß, (2012), arXiv:1211.0289.

[88] P. Fileviez Perez and S. Spinner, (2012), arXiv:1209.5769.

[89] S. King, Phys.Lett. B659, 244 (2008), arXiv:0710.0530. 
[90] F. del Aguila et al., Eur.Phys.J. C57, 183 (2008), arXiv:0801.1800.

[91] F. Iocco, G. Mangano, G. Miele, O. Pisanti, and P. D. Serpico, Phys.Rept. 472, 1 (2009), arXiv:0809.0631.

[92] Particle Data Group, J. Beringer et al., Phys.Rev. D86, 010001 (2012).

[93] E. Nardi and E. Roulet, Phys.Lett. B245, 105 (1990).

[94] C. F. Berger, L. Covi, S. Kraml, and F. Palorini, JCAP 0810, 005 (2008), arXiv:0807.0211.

[95] M. Malinsky, Phys.Rev. D77, 055016 (2008), arXiv:0710.0581.

[96] R. Slansky, Phys.Rept. 79, 1 (1981).

[97] J. Kopp, M. Lindner, V. Niro, and T. E. Underwood, Phys.Rev. D81, 025008 (2010), arXiv:0909.2653. 